\title{
Design and synthesis of novel thiazole CYP51 inhibitors as therapeutics for Candida Albicans infections
}

Marwa Alsulaimany ${ }^{\mathrm{a}}$, Josie E. Parker ${ }^{\mathrm{b}}$, Andrew G. Warrilow ${ }^{\mathrm{b}}$, Steven L. Kelly ${ }^{\mathrm{b}}$, Diane E. Kelly and Claire Simons ${ }^{\mathrm{a}}$

aSchool of Pharmacy and Pharmaceutical Sciences, Cardiff University, King Edward VII avenue, Cardiff, CF10 3NB, UK; ${ }^{b}$ Centre for Cytochrome P450 Biodiversity, Institute of Life Science, Swansea University Medical School, Singleton Park, Swansea, SA2 8PP, UK.

Lanosterol $14 \alpha$-demethylase, CYP51, is an important target enzyme in fungal diseases. Candida albicans (C. albicans) is one of the fungal pathogens prevalent in human infection, which causes infections that range from superficial to life-threatening systemic infections, a particular challenge in immunocompromised patients. Although azoles (especially Fluconazole) have been used as a first choice for the treatment in many fungal infections and as a prophylactic, issues of drug resistance to this class of antifungals are increasing. The aim of this study was to design and synthesis novel CYP51 inhibitors that can counteract the azole resistance mechanism in C.albicans and importantly show selectivity for CaCYP51 over human CYP51 and endogenous CYPs to avoid side effects and toxicity.

Drug design employed computational methods (Molecular Operating Environment (MOE) for docking and binding studies and Desmond Maestro for MD simulations) to determine optimal fit in the CaCYP51 active site and binding interactions. From the computational docking study, two series of thiazole derivatives were selected for synthesis; a short series (fluconazole type) and an extended series (posaconazole type). The extended series was observed to have optimal filling of the active site and additional binding interactions (H-bonding and $\pi$ - $\pi$ /hydrophobic) that were anticipated to counteract the loss of one key H-bonding interaction with Tyr132, a common mutation in C.albicans azole resistant strains (Y132H, Y132F). Using a 4-6 synthetic scheme, both series were obtained and all compounds subject to structure $\left({ }^{1} \mathrm{H}\right.$ and ${ }^{13} \mathrm{C}$ NMR, mass spectroscopy) and purity (HPLC) analysis.

All final compounds were evaluated against Candida strains (MIC) and promising compounds evaluated further for CaCYP51 binding affinity $\left(K_{d}\right)$ and inhibitory activity $\left(\mathrm{IC}_{50}\right)$ in comparison with the standards fluconazole and posaconazole. Those compounds with optimal activity will be further evaluated for selectivity against human CYP51 and sterol profile and this data will be presented.

Claire Simons, School of Pharmacy and Pharmaceutical Sciences, Cardiff University, simonsc@,cardiff.ac.uk

Marwa Alsulaimany, School of Pharmacy and Pharmaceutical Sciences, Cardiff University, alsulaimanymn@cardiff.ac.uk 
Josie E. Parker, Centre for Cytochrome P450 Biodiversity, Institute of Life Science, Swansea University Medical School, j.e.parker@swansea.ac.uk

Andrew G. Warrilow, Centre for Cytochrome P450 Biodiversity, Institute of Life Science, Swansea University Medical School, a.warrilow@swansea.ac.uk

Steven L. Kelly, Centre for Cytochrome P450 Biodiversity, Institute of Life Science, Swansea University Medical School, s.l.kelly@swansea.ac.uk

Diane E. Kelly, Centre for Cytochrome P450 Biodiversity, Institute of Life Science, Swansea University Medical School, d.kelly@swansea.ac.uk 